\title{
Analysis of controversy late deceleration in fetal heart rate monitoring
}

\author{
Kazuo Maeda* \\ Department of Obstetrics and Gynecology, Emeritus, Tottori university, Medical School, Yonago, Japan
}

\begin{abstract}
Developmental process of late deceleration (LD) of fetal heart rate (FHR) was analyzed to clarify controversy LD record. The mechanical compression of pelvic large vessels by contracted uterine body develops $\mathrm{LD}$, and fetal hypoxia will be enhanced to severe level. Ppathologic LD should be searched. The loss of variability associated with LD was severe pathology. Actocardiogram, is useful to predict fetal outcome in LD cases.
\end{abstract}

\section{Introduction}

Transient FHR reduction (deceleration) was classified to $U$ shaped variable one (VD) and $\mathrm{V}$ shaped periodic one, which was divided into early deceleration (ED) and late deceleration (LD), of which delay to uterine contraction is 20 or more S(Figure 1). LD was placental insufficiency. and its outcome was ominous [1]. Caldeyro- Barcia studied Type I dip, which was the same as ED, and Type 2 dip was $\mathrm{LD}$ [2].Some cases, who were diagnosed as $\mathrm{LD}$, received cesarean (C-) sectionaccording to FHR pattern theory expecting depressed neonate, while theneonate was vigorous, confusing doctor by the paradoxical results. Therefore, the author intended to study the mechanism to develop the LDin past reports [2-4]. The principle of some LDs was the compression of maternal pelvic lrge vessels with contracted uterus prior to the FHRdeceleration with lag time, while it disappeared by the change of maternal posture to lateral one [3]

\section{Methods}

The situations to develop delayed FHR deceleration after uterine contraction were searched in past literatures [1-4].

\section{Results}

\section{Compression of large maternal pelvic blood vessels:}

1. Supine hypotension: Inferior vena cava was compressed by contracted pregnant uterus reducing the bloodreturn to maternal heart and developed maternal hypotension followed by the reduction of placental arterial blood flow, developing hypoxia and delayed deceleration in FHR record. It is well known that the $\mathrm{LD}$ and supine hypotension disappear after changing maternal posture to lateral one from the supine, and also the deceleration adisappears.However, the mechanism to delayed development to the contraction is hardly explained.

2. Pelvic large iliac arteries were compressed by contracted pregnantuterus in the labor at maternal supine posture, stopping placental arterial blood supply, developing fetal hypoxia and FHR deceleration after the contraction (Poseiro effect). The loss of iliac arterial flow due to the compression was confirmed by pelvic angiography. The LD, FHR deceleration after uterine contraction, disappeared after changing maternal posture to lateral from supine one [3]. The late appearance to uterine contraction and its disappearance after lateral posture are clearly explained, because vascular compression developed after uterine contraction

Fetal damage in repeated hypoxia parallel to the bradycardia in $\mathrm{LD}$

As the bradycardia of LD cause by iliac artery compression with contracted uterine body was about $100 \mathrm{bpm}$ at each nadia in Figure 1 , thus fetal hypoxia, which is parallel to bradycardia,was mild, not to reach prompt fetal damage in early stage of $\mathrm{LD}$, thus, the neonate delivered in this stage is vigorous.

Hypoxic fetal deterioration will accumulate when the $\mathrm{LD}$ repeated for long period, for example, the mother refused C-section, LD repeated in $50 \mathrm{~min}$, where fetal damage accumulated to the high level of the loss of variability (Figure 1). The result was severe neonatal depression, Apgar score was 3, and the baby died in 3 month due to rain hemorrhage. This will be ominous type LD.

The strategy to avoid this severe LD will be the treatment in early stage by changing from supine posture, and the C-section is no effect in early stage treatment, where developmental mechanism should be clarified.

Severe hypoxic changes developed some weeks after early fetal hypoxia in the loss of FHR acceleration in fetal growth restriction (FGR). The severe hypoxia was LD, fetal bradycardia and the loss of variability, resulting heavy neonatal asphyxia and neonatal deaths [5], where the $\mathrm{LD}$ was pathologic, therefore, early delivery was recommended before the loss of acceleration in FGR [5].

Correspondence to: Kazuo Maeda, Department of Obstetrics and Gynecology, Emeritus, Tottori university 3-125, Nadamachi, Yonago, Tottoriken, 683-0835 Japan, Phone and FAX; 81-859-22-6856; E-mail; maedak@mocha.ocn.ne.jp

Key words: fetal heart rate, late deceleration, Large vessel compression, the loss of variability, fetal growth restriction, hypoxia index, actocardiogram

Received: April 28, 2017; Accepted: May 22, 2017; Published: May 25, 2017 


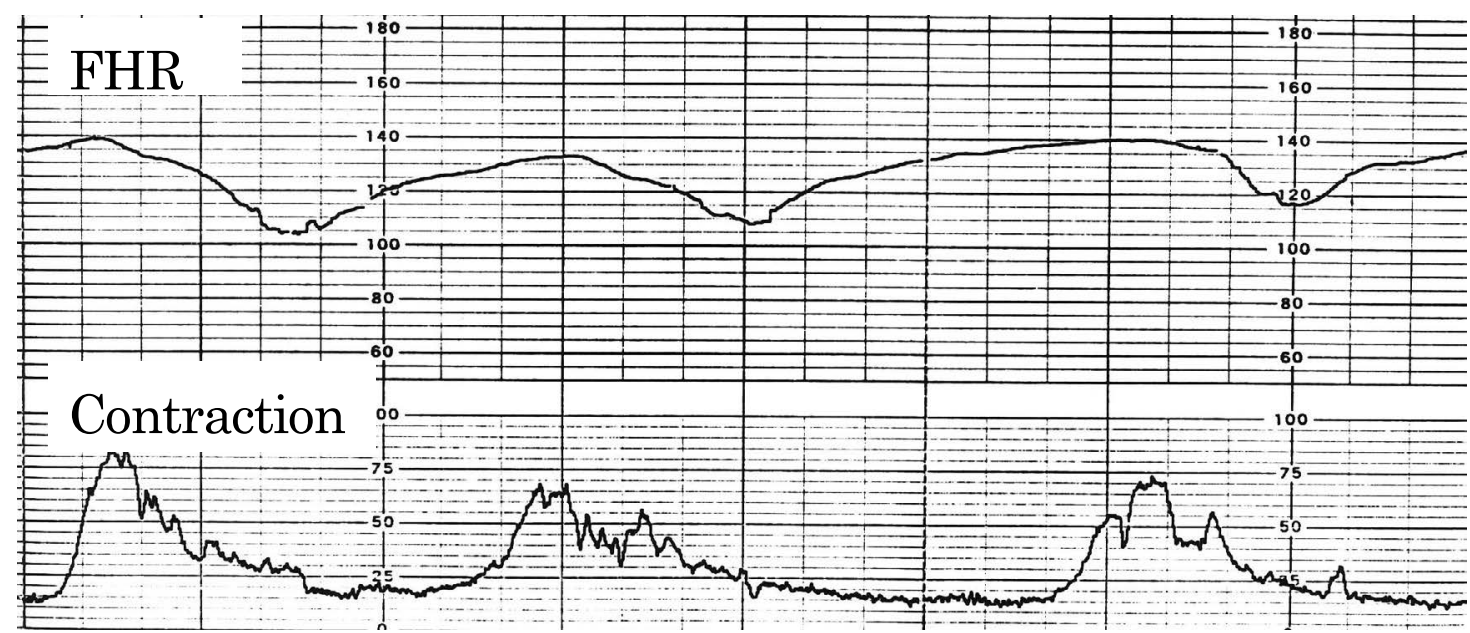

Figure 1. Typical LD and the loss of variability in the labor. Fetal hypoxia index was 25. One min Apgar score was 3 . Three month infant died due to brain hemorrhage

\section{The loss of FHR variability}

Fetal outcome was ominous even in very mild LD by Hon [1], however, mild LD was recognizable when FHR variability is lost [1]. As the loss of variability was severe fetal brain damage similar to anencephaly [6], fetal asphyxia is treated by the C-section before the loss of variability

\section{Discussion}

\section{Causes of hypoxic FHR deceleration}

1. Fetal disordersCongenital or acquired fetal diseases develop fetal hypoxia and FHR deceleration, because the heart rate is parallel to $\mathrm{PaO}_{2}$ when il is lower than $50 \mathrm{mmHg}$, which is common level of fetal arterial blood.

2. Extrinsic causesLow maternal blood pressure, maternal hypoxia, abnormal placental oxygen transfer function, e.g. infarction in preeclampsia, intervillous space fibrin deposit, umbilical cord compression or uterine hypercontraction.

\section{Compression of maternal pelvic vessels}

\section{a. Supine hypotension of mother}

Maternal inferior vena cava is compressed by contracted pregnant uterus in maternal supine posture causing maternal hypotension by reduced blood return to the heart and fetal hypoxia causing late deceleration, which disappears after change of maternal posture to lateral posture.

\section{b. Compression of iliac arteries}

Contracted pregnant uterus in maternal supine posture stopps the arterial blood supply to the placenta causing fetal hypoxia. The mechanism was found by pelvic angiography (Poseiro effect) [3]. Fetal deceleration occurs after the lag time, namely, late deceleration (LD) appears, where the LD disappears after maternal posture change to lateral one [3].

\section{c. Other Causes}

Umbilical cord compression, placental insufficiency, etc.

\section{Management of late deceleration}

Asmaternal lateral posture rejects the pelvic vessel compression caused by contracted pregnant uterus in maternal supine posture, i.e., the vessel compression is removed by maternal lateral posture increasing the blood supply to the placenta. As the effect of lateral posture is revealed by quick disappearance of $L D$ [4], the mother has to take lateral posture immediately after recording LD in fetal monitoring to confirm whether the cause of $\mathrm{LD}$ is compressed pelvic large vessel or not, i.e. it should be known that the LD is caused by vessel compression with the contracted uterus, while staffs have to further search LD developing pathologic cause, if LD remains on FHR record after the change to lateral posture.

\section{Tocolysis will be done in the uterine hypercontraction}

Orcinoprenaline was effective to the LD in uterine hyper contraction inthe past [4].. The tocolysis will be effective by the terbutaliine at present.

\section{Early delivery}

Severe LD case is cured by early delivery with C-section before the loss of variability preventing fetal brain damage, when intrauterine therapy was ineffective. Timely $\mathrm{C}$-section will be performed before the loss of FHR variability, which is the sign of heavy fetal brain damage resulting neurological sequels, e.g. cerebralpalsy [6]. Timely C-section will be indicated by severe FHR changes, the loss of FHR acceleration, decreased variability [6], or when the hypoxia index was 20-24, as the index of cases of the loss of variability was $25-26$. The index is determind by the sum of FHR bradycadia duration ( $\mathrm{min}$ ) x100, divided by the lowest bradycardia (bpm), because FHR lower than $110 \mathrm{bpm}$ will be parallel to $\mathrm{PaO}_{2}$ lower than $50 \mathrm{mmHg}$..

\section{Actocardiogram}

The neonate was vigorous when fetal acceleration duration ratio to fetal movement burst duration $(\mathrm{A} / \mathrm{B}$ ratio) was higher than 1.0 , despite repeated LDs were recorded, namely, fetal movement study was effective to estimate favorable outcome even in the presence of LD [7].

\section{Possibility of fetal/neonatal damage}

Although FHR is parallel to the $\mathrm{PaO} 2$ under $50 \mathrm{mmHg}$, where fetal bradycardia is recorded in monitoring chart, it is not the same as fetal damage, namely, the bradycardia is caused by the neurologic excitation of parasympathicus center located in the medulla oblongata, and the experimental hypoxic bradycardia disappears after the narcosis of rabbit, [8], and the apneic bradycrdia of anenephalic newborn, who remains medulla oblongata, disappeared by the infusion of oxygenated blood into the vessel of anencephalic neonate [9]. 
Fetal brain is damaged when FHR acceleration is lost and the variability lost by the hypoxia, namely fetal bradycardia shows only fetal hypoxic environment, while hypoxic fetal brain damage is expressed by the loss of fetal brain response to fetal movement, i.e. FHR acceleration and the variability are fetal brain response to fetal movement burst and minor fetal movements, therefore, the loss of acceleration to fetal movement is is early sign of fetal brain damage, while the loss of variability is advanced severe fetal brain damage, confirmed by actocardiographic studies [6,7].

Therefore, the early appearance of LD caused by the compression of Large iliac vessels which recovered after posture change can not be fetal damage, therefore, the neonate is vigorous without brain damage, while sum of hypoxic effects in repeated LDs for several 10 min may cause fetal brain damage.

The fetal damage could be estimated by the hypoxia index, which is the sum of duration of fetal bradycardia ( $\mathrm{min}$ ), multiplied by 100 , and divided by the lowest FHR (bpm). As the Index after the loss of viability was $25-26$, it must be around 20 to prevent fetal brain damage. However, the author hopes rapid cure of LD case in its earliest stage, that is, the cure of $\mathrm{LD}$ caused by uterine compression by the change to lateral posture. Actocardiographic A/B ratio will show correct outcome.

\section{Conclusion}

There would be some kinds of LD, including simple maternal iliac artery compression by contracted uterine body, whose outcome is favorable after early maternal lateral posture, and hypoxic fetal damage will appear in long repeated LD. Although final decision will be C-section, early maternal lateral posture would be tried, where outcome will be favorable. The actocardiogram will predict outcome by it's a/B ratio. The hypoxia index should be less than 20 , to expect no fetal brain damage.

\section{References}

1. Hon EH (1968) An atlas of fetal heart rate patterns. Harty Press.

2. Caldeyro-Barcia R, Poseiro JJ, Mendez-Bauer C, Gulin LO (1967) Effects of Abnormal Uterine Contractions on Fetal Heart Rate in Labor. 9-27.

3. Poseiro JJ, Mendez-Bauer C, Caldeyro-Barcia R et al. (1969) Effect of uterine contractions on maternal blood flow through the placenta. Perinatal factors affecting human development, Paho Advisary Committee. 161-171.

4. Caldeyro-Barcia R, Magana JM, Poseiro JJ, et al. (1969) New Approach tothe treatment of acute Intrapartum fetal distress. Perinatal factorsaffecting human development. Patho Advisary Committee, 248-253.

5. Teshima N (1993) Non-reactive pattern diagnosed by ultrasonic Doppler fetal actocardiogram and outcome of the fetuses with non-reactive pattern. Acta Obstet Gynecol Jpn 45: 423-430.[Crossref]

6. Maeda K (2014) Modalities of fetal evaluation to detect fetal compromise prior to the development of significant neurological damage. J Obstet Gynaecol Res 40: 20892094.[Crossref]

7. Maeda K (2016) Fetal Actocardiogram. Analysis of Fetal Motion and Heart Rate, Jaypee Brothers, New Delhi, 2016.

Copyright: (C2017 Maeda K. This is an open-access article distributed under the terms of the Creative Commons Attribution License, which permits unrestricted use, distribution, and reproduction in any medium, provided the original author and source are credited. 